\title{
Cognitive Radio Spectrum Sensing Mechanisms in TV White Spaces: A Survey
}

\author{
Kenneth Kimani \\ Department of Telecommunications \& Information \\ Engineering, Jomo Kenyatta University of Agriculture and \\ Technology, Juja, Kenya \\ kuriakimani@yahoo.com
}

\author{
Morris Njiraine \\ Department of Telecommunications \& Information \\ Engineering, Jomo Kenyatta University of Agriculture and \\ Technology, Juja, Kenya \\ morrisnjiraine@gmail.com
}

\begin{abstract}
Frequency spectrum is a limited resource and the increasing demand caused by emerging services, augmented number of wireless users along with the demand for high-quality multimedia applications have resulted in the overcrowding of the allocated spectrum bands. The overcrowding of spectrum bands has been exacerbated by the current spectrum licensing policy which has emerged as a bottleneck to efficient spectrum utilization, due to its inflexibility, resulting in most of the licensed spectrum being severely under-utilized. However, the problem of scarcity of spectrum bands and the inefficient utilization of the already allocated radio spectrum can be smartly addressed through spectrum sharing by enabling opportunistic usage of the underutilized frequency bands. One of the most exciting ways of spectrum sharing is cognitive radio technology which allows a wireless node to sense the environment, detect the network changes, and then make intelligent decisions by dynamically changing its reception or transmission parameters to communicate while ensuring that no interference is affected to the licensed users. It thus improves the spectrum utilization by reusing the unused or underutilized spectrum owned by the incumbent systems (primary systems). In this paper, a comprehensive survey and review of recent research about the advances in cognitive radio technology will be carried out. We will also evaluate the various spectrum sensing techniques in a cognitive radio network in the UHF/VHF bands allocated for TV broadcasting.
\end{abstract}

Keywords-cognitive radio; spectrum sensing techniques; opportunistic spectrum access;

\section{INTRODUCTION}

The rapid growth of wireless communication has exacerbated the problem of scarcity of available spectrum. Since the bulk of the spectrum has already been assigned, it is extremely arduous to find new spectrum for either deploying new services or expanding already existing ones. Although nearly all of the spectrum bands have already been allocated, these bands are seriously underutilized most of the time. The traditional practice of clearing and then reassigning portions of the allocated spectrum is not a sustainable model as it is both time-consuming and expensive. However, spectrum sharing is possible in order to better utilize the spectrum and make current wireless systems more efficient. Spectral underutilization can be enhanced by permitting a cognitive user to access a licensed band when it is idle. Efficient spectrum utilization is the most challenging hurdle in the evolution of wireless communication systems and radio devices. Cognitive radios (CRs) have emerged as a solution through opportunistic spectrum sharing of spatially and temporally unused spectrum bands. This helps to improve spectral utilization by reusing the unused or underutilized spectrum owned by incumbent systems (primary systems). One of the most fundamental aspects of a CR radio is spectrum sensing as it enables a secondary user to detect the parts of a spectrum band that are currently underutilized and unutilized in real-time. Spectrum sensing algorithms are used to obtain awareness about spectrum occupancy and the existence of primary users in a given spectrum band. This allows spectrum sharing and gives opportunistic access to unused spectrum and hence enhances spectrum utilization. Use of spectrum sharing instead of exclusivity has created new opportunities for new technologies such as a smart grid communication network.

\section{COGNITIVE RADIO TECHNOLOGY}

CR devices are intelligent and self-conscious devices that can observe and detect changing environmental conditions and adjust their operating parameters like power, frequency, coding and modulation techniques, according to the changing communication environment culminating in efficient utilization of available resources [1]. Spectrum scarcity and inefficient spectrum utilization are some of the most challenging issues in wireless communications. $\mathrm{CR}$ provides an answer to the spectral dearth by enabling the opportunistic exploitation of frequency bands that are not densely occupied by licensed users. This allows secondary users to utilize them momentarily hence enhancing utilization. By enabling opportunistic spectrum access (OSA), CR helps mitigate the spectrum scarcity issue by allowing secondary devices to establish the un-/underutilized sections of licensed spectrum and exploit them opportunistically as long as no harmful interference is caused to the legacy spectrum users' communications. The provisionally unutilized parts of spectrum are referred to as spectrum white spaces (WS) or spectrum holes and they may exist in time, frequency, or space domains. In the context of OSA, CR users are referred to as secondary users (SUs) in contrast to the primary users (PUs). CRs enable vacant 
spectrum detection and spectrum sharing for a more efficient spectrum utilization.

\section{OPPORTUNISTIC SPECTRUM ACCESS}

$\mathrm{CR}$ enables the interim exploitation of unused spectrum. This allows the coexistence of two different systems within the same frequency range: the primary system and the secondary system. The primary system refers to the licensed users that have exclusive rights to make use of the assigned spectrum. The secondary system is the unlicensed CR users that do not own any rights to the spectrum but can access the spectrum momentarily as long as it is not used by the primary system [2]. In case the licensed user requires to use the spectrum, the CR vacates to another spectrum hole or remains in the same band, but alters its parameters like the power level or modulation scheme to avoid interference [3, 4]. The main reasons for spectrum underutilization are:

1. The spectrum occupancy ratio is higher during the day than during the night. This leads to underutilization of spectrum especially at night.

2. The presence of guard bands in the spectrum intended to safeguard against adjacent channel interferences between neighboring channels especially in the analogue TV broadcasts.

IEEE 802.22, is a wireless standard designed to utilize CR for regional area networks in TV white spaces by enabling the sharing of geographically unused television spectrum while ensuring that no interference is occasioned to the licensed PUs. PUs in this case includes both analog and digital TV transmissions, and licensed low power devices such as wireless microphones [5].

\section{MAJOR FUNCTIONS OF COGNITIVE RADIO}

There are four main steps in the cognitive cycle:

\section{A. Spectrum Sensing}

This refers to the detection of the unused spectrum portions that can be exploited without causing harmful interference to other users [6]. It is a vital requirement for the SUs to identify the spectrum holes and is usually the first step of the cognitive cycle.

\section{B. Spectrum Management}

It is the process of detecting the best available spectrum to meet the cognitive user communication requirements. Keeping track of the next available frequency band allows the SUs to hop between multiple frequency bands in response to time varying channel characteristics or in response to the reappearance of the PU while still maintaining acceptable Quality of Service (QoS) requirements. The spectrum management functions are categorized as:

- Spectrum analysis: In spectrum analysis, spectrum sensing results are examined to evaluate the available spectrum. This involves measuring the quality of spectrum accessed by a SU. This quality can be evaluated using the average correlation, Signal to Noise Ratio (SNR) and white spaces availability.
- Spectrum Decision: Before the opportunistic spectrum access occurs, a decision model should be adopted. The decision model provides guidance on the rules and priority mechanisms to be followed in spectrum accessing. The complexity of such a model is dependent on the parameters to be considered in the spectral analysis (transmission bandwidth, data rate, transmission mode, etc. [7]). When both SUs and PUs are present in the system, a preference mechanism is required in order to determine the priority of spectrum access. SUs can be cooperative or noncooperative. In a cooperative environment, the secondary CRs collaborate with each other, to make a collective decision regarding the vacancy of a given frequency band. In that case, a central controller coordinates the spectrum management process [7].

\section{Spectrum Mobility:}

This refers to the changeover of operating frequency band of CR users from one spectrum hole to another. When a PU reappears in a radio channel which is currently in use by a SU, the cognitive user has to change its frequency of operation. This switch in operating frequency band is referred to as spectrum handoff [8]. It ensures that the unlicensed SU continues with uninterrupted data transmission even after switching to the new band [8] by occupying the best available spectrum hole in order to meet user communication requirements.

\section{Spectrum Sharing}

This refers to the provision of a fair spectrum scheduling algorithm among the different users. Since there might be many different SUs who want to exploit the available spectrum holes at any given time, CR has to enforce policy rules to maintain fair spectrum scheduling among the different cognitive and non-cognitive users in the radio environment with a view to creating a balance between the goals of efficient information exchange and of sharing the available spectrum [7]. Although dynamic spectrum access is championed as a solution to the current static allocation of spectrum due to its flexibility and high spectrum utilization, spectrum sharing is still a major challenge in an open spectrum usage environment due to the existence of some greedy SUs who might not want to cede spectrum to other cognitive users. It provides a balance and fair spectrum scheduling method between coexisting SUs and PUs [3].

\section{SPECTRUM SENSING CLASSIFICATION}

Sensing algorithms can be classified into three major categories: transmitter detection, cooperative sensing and interference based sensing. Transmitter detection techniques are further categorized into energy detection, matched filter detection and cyclostationary feature detection (Figure 1).

\section{SPECTRUM SENSING TECHNIQUES}

\section{A. Transmitter Detection}

Spectrum sensing is the cornerstone of a successful CR based communication as it determines the occupancy status of a spectrum band by periodically sensing the target frequency band. Once a CR has detected a spectrum hole in frequency, 
space or time domain, it has to also determine the access method in terms of transmit power to employ and access duration in order to transmit without interfering with a licensed user's transmission $[6,9]$.

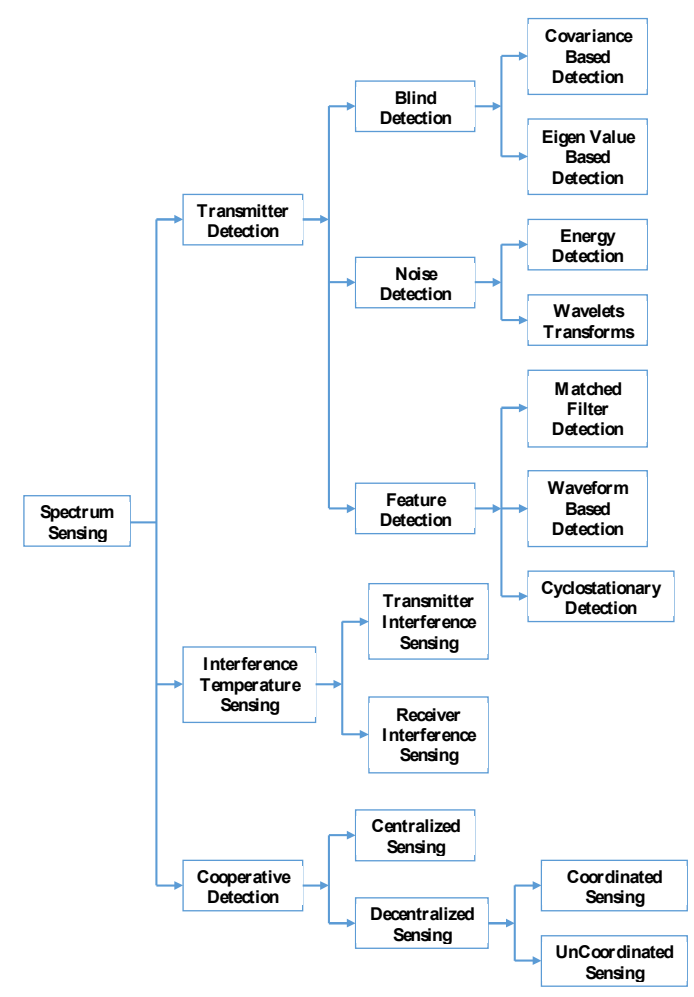

Fig. 1. Classification of spectrum sensing techniques

Although there are various techniques for establishing the presence of a PU signal transmission, the most effective way is to sense spectrum holes by detecting active primary transceivers in the vicinity of the cognitive radios. This is referred to as transmitter detection and can broadly be categorized into three main groupings, depending on the type of information required for the sensing operation:

- Noise dependent detection: These detection algorithms only require information on noise properties and do not need to make any assumptions on the primary signal characteristics. They do not need any prior knowledge of the signal but they do need very accurate information on noise statistics in order to obtain reliable detection performance.

- Feature detection: These detection algorithms require prior knowledge of both source signal and noise power characteristics. They then employ knowledge of the structural and statistical properties of PU signals to make a decision on the presence or absence of a source signal.

- Blind detection: These detection algorithms rely on statistical analysis to identify the properties of a signal. These detection mechanisms detect the primary signal by sensing the surrounding environment without requiring any preliminary information of the noise power or the source signal.

\section{1) Various Transmitter Detection Techniques}

\section{a) Matched Filter Detection}

A matched filter (MF) is a linear filter that aims at maximizing the output SNR for a given input signal. This is a detection method that relies on prior knowledge of the PU signal, e.g. the modulation scheme, the pulse shaping, and the packet format. Using this information, a SU can correlate the detected signal with a corresponding PU signal, and then sample the output to determine if the detected signal is the PU signal $[10,11]$. MF operation can be equated to correlation whereby an undetermined signal is convolved with a filter whose impulse response is a mirror and time shifted version of a reference (PU) signal [10]. It is therefore a feature detection algorithm. Mathematically, matched filter operation can be denoted as:

$$
Y(n)=\sum_{k=-\infty}^{\infty} h(n-k) x(k)
$$

where $Y(n)$ is the output of the matched filter, given that $x(n)$ is the received signal and $h(n)$ is the filter response. Its block diagram is shown in Figure 2.

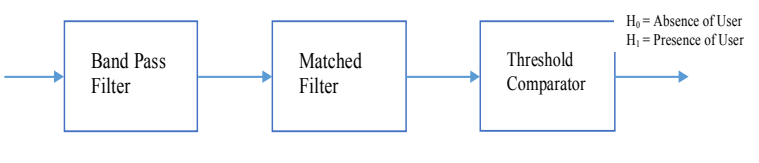

Fig. 2. Block diagram for MF detection

\section{b) Energy Detection}

This detection method requires no prior information of the PU signal. It is a non-coherent method that detects the primary signal solely based on the sensed energy. PU is detected based on the sensed energy where the received signal energy is computed and compared against a pre-defined threshold. If the measured value surpasses the threshold, then the received signal is deduced to be a PU signal, and the spectrum band is assumed to be occupied. Else, the spectrum band is inferred to be idle [12]. Energy detection (ED) is categorized as a noise dependent signal detector since it approximates the presence of a PU signal by comparing the sensed energy with a known threshold derived from the noise statistics [11, 13, 14]. Mathematically, signal detection can be simplified to a simple identification problem, defined as a hypothesis test:

$$
\begin{aligned}
& x(n)=w(n) \quad \mathrm{H}_{0} \text { (PU absent) } \\
& x(n)=h * s(n)+w(n) \quad \mathrm{H}_{1} \text { (PU present) }
\end{aligned}
$$

where $s(n)$ is the signal transmitted by the PUs, $x(n)$ is the signal received by the $\mathrm{SU}, w(n)$ is additive white Gaussian noise (AWGN), and $h$ is the amplitude gain of the channel. The estimated energy of the received signal can be expressed mathematical as:

$\sum_{n=0}^{N}|x(n)|^{2}$

The energy can now be compared to the threshold to check the true hypothesis by using:

$$
\begin{aligned}
& \mathrm{H}_{1} \text { if } \in>\lambda \\
& \mathrm{H}_{0} \text { if } \in<\lambda
\end{aligned}
$$


The steps in an energy detector are illustrated in Figure 3.

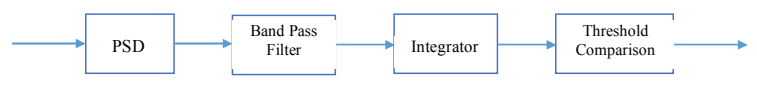

Fig. 3. Energy detector block diagram

\section{c) Cyclostationary Feature Detection}

Cyclostationary feature detection capitalizes on the periodicity in the received primary signal to determine the presence of a PU. Most of the transmitted signals from the PUs usually have periodic patterns. These periodic patterns are known as cyclostationarity and are used to detect the presence of licensed users. The periodicity is usually found in sinusoidal carriers, pulse trains, spreading code, hopping sequences or cyclic prefixes of the primary signals which results in periodicity of their statistics like mean and auto correlation [11]. When the cyclic spectral density (CSD) of such signals is computed, it helps in highlighting such periodicities. Due to cyclostationarity, primary signals can easily be identified since the features of periodic statistics and spectral correlation, are normally absent in stationary noise and in interference. This helps in distinguishing PUs from noise. Thus, prior knowledge of the signal features is necessary in cyclostationary feature detection. This method detects even in very low SNR as the cyclostationarity embedded in the PU signal is non-existent in noise [13]. Cyclostationary detection can be illustrated as shown in Figure 4.

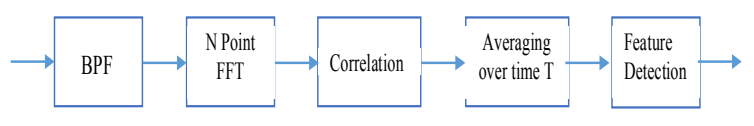

Fig. 4. Block diagram for cyclostationary feature detection

A received signal $x(t)$ is said to be cyclostationary if its mean and auto correlation shows periodicity as [15]:

$$
\begin{aligned}
& m_{x}\left(t+T_{0}\right)=m_{x(t)} \\
& R_{x}\left(t+T_{0}, u+T_{0}\right)=R_{x}(t, u)
\end{aligned}
$$

where the period of mean and auto correlation is $T_{0}$. If $t$ and $u$ are substituted in the autocorrelation equation with $t+\tau / 2$ and $t$ $\tau / 2$, then:

$$
R_{x}(t+\tau / 2, t-\tau / 2)=\sum R_{x}^{\alpha}(\tau) e^{j 2 \pi \alpha t}
$$

where $R_{x}^{\alpha}$ depicts the cyclic autocorrelation function (CAF) and $\alpha$ symbolizes the cyclic frequency. Cyclic frequency is assumed to be a known parameter to the receiver. CAF is computed as:

$R_{x}^{\alpha}(\tau)=\lim _{T \rightarrow \infty} \frac{1}{T} \int_{-1 / T}^{1 / T} R_{x}(t+\tau / 2, t-\tau / 2) e^{-j 2 \pi \alpha t} d t(10)$

Cyclic spectral density (CSD) is obtained as:

$$
S_{x}^{\infty}(f)=\int_{-\alpha}^{\alpha} R_{x}^{\alpha}(\tau) e^{-\mathrm{j} 2 \pi \int \tau} d \tau
$$

where $R_{x}^{\alpha}(\tau)$ is the cyclic autocorrelation function (CAF).

$$
\text { d) Wavelet Detection }
$$

The wavelet based detection method uses wavelets to detect edges in the power spectral density (PSD) of a wideband channel [16] as shown in Figure 5.

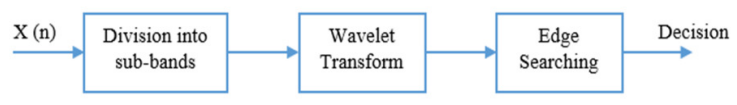

Fig. 5. Block diagram for wavelet based sensing

This approach breaks down the wide spectrum into small non-overlapping sub-bands and tries to identify the variations in the power level in these sub bands. The breakdown is accomplished by using wavelet transform which identifies the edges in PSD. These edges in PSD mark the boundary between occupied bands and spectrum holes thus assist in identifying the vacant bands. When these edges of sub bands are detected, the powers between two edges can be estimated [17]. This helps in determining whether the band is occupied or not. Using this information, CR users can ascertain spectrum holes and exploit them [18]. It is a flexible and low cost blind detection technique $[19,20]$.

\section{e) Covariance Based Sensing Technique}

Covariance based detection (CBD) utilizes covariance matrixes for detection since the statistical covariance matrixes of noise are quite different from the ones of a received signal. This property is therefore used to distinguish if a licensed $\mathrm{PU}$ is present or absent in a channel. This technique uses the correlative nature of a received signal, to compute the covariance matrix of the detected signal samples and then compares it with the covariance of noise to determine if a primary signal is present $[19,21]$. Autocorrelation can also be used to compute the thresholds in place of covariance [17]. This approach does not need any previous information of the primary signal and works well for highly correlated signals. It has a very high probability of differentiating between the signal and noise even at extremely low SNR values. It also has very low power consumption. Its main weaknesses are the increased complexity and the increased computational overhead. Since it needs no prior information on the primary signal, it is referred to as a low power blind detection technique. Its block diagram is shown in Figure 6.

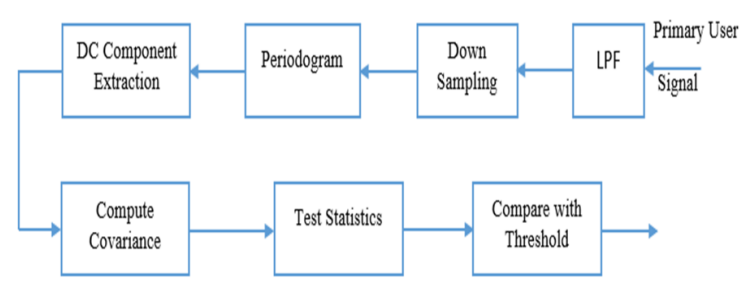

Fig. 6. Block diagram for covariance based detection

\section{f) Radio Identification Based Sensing.}

In radio identification technique, several features such as modulation, transmission range, transmission frequency, etc., are extracted from the received signal and they are then used to decide the most appropriate technology for the CR transmission. This approach tries to detect the presence of some 
known technology being used by the sensed primary signal such that if the technology used by the PU for transmission is known, then its spectrum characteristics can be inferred [17]. $\mathrm{CR}$ users can then use this information to alter their transmission parameters in order to achieve communication [18]. This detection method is highly accurate, is robust to SNR and has average sensing time. However, it has high power consumption due to its high complexity.

\section{g) Random Hough Transform Based Detection}

Hough transform is a technique that is widely used for pattern detection in image processing [18]. Random Hough transform of the detected signal is used to search for the presence of radar pulses in the operating channels of WLAN systems. If some patterns related to PUs are identified, CR users can presume that a PU is using the spectrum while the spectrum will be assumed to be idle if patterns related to the primary signal are not identified. This technique is ideal for observing signals with periodic patterns [17].

\section{h) Eigenvalue Based Sensing Technique}

In eigenvalue sensing, the PU signal is identified by calculating the eigenvalues of the covariance matrix of the received signal [19]. This method utilizes the received signal samples and requires no prior knowledge of the signal or the channel hence eliminating any need for synchronization. Eigenvalue based sensing can be categorized into two main approaches:

- Maximum-minimum eigenvalue detection (MME): In this approach, the ratio of maximum to minimum eigenvalue is used to identify the primary signal by employing random matrix theories (RMT). This ratio is then quantized to find the threshold value.

- Energy with minimum eigenvalue (EME) based detection: In EME, detection is carried out using the ratio of the signal energy to the minimum eigenvalue. EME compares the received signal energy with the minimum eigenvalues of the sample covariance matrix of the received signal.

\section{i) Waveform-Based Detection}

This method takes advantage of the fact that known patterns are usually employed in wireless networks for synchronization purposes. Such patterns corresponding to the signal, such as preambles, mid-ambles, regularly transmitted pilot patterns and spreading sequences are utilized to detect the signal presence. Preambles are sets of patterns that are transmitted just before the start of the data transmission sequence whereas mid-ambles are transmitted in the median of the data sequence. These patterns are mostly used for synchronization purposes. Therefore, if a known pattern of the signal is present, this detection method can be applied by correlating the received signal with a known copy of itself $[18,22]$. The longer the length of these known patterns, the more accurate the detection. This is therefore a feature detection algorithm. For a received signal $y(n)=s(n)+w(n)$, the waveform based sensing can be expressed as:

$$
\mathrm{M}=\mathcal{R} e\left[\sum_{n=1}^{N} y(n) s^{\prime}(n)\right]
$$

In absence of a PU, the waveform based detection can be represented as:

$$
\mathrm{M}=\mathcal{R} e\left[\sum_{n=1}^{N} w(n) s^{\prime}(n)\right]
$$

In presence of a PU, (13) becomes:

$$
\mathrm{M}=\sum_{n=1}^{N}|s(n)|^{2}+\mathcal{R} e\left[\sum_{n=1}^{N} w(n) s^{\prime}(n)\right]
$$

The presence or absence of a PU is determined by comparison of the decision metric $\mathrm{M}$ against a threshold value $\lambda_{W}$ as shown in Figure 7.

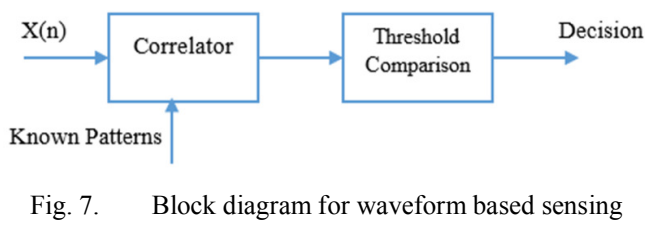

\section{2) Comparison of Spectrum Sensing Techniques}

ED based spectrum sensing is the most popular spectrum sensing algorithm due to its low computation and implementation complexity. It requires no prior information of the PU signal resulting in short sensing time. Its main disadvantage however, is that it does not work very well in low SNR conditions which at times results in high probability of false alarm and miss-detection[11, 23]. MF detection on the other hand, has a very small observation period since a high processing gain can be achieved by coherent detection. Its major drawback however, is that each CR requires a dedicated receiver for every PU class and prior knowledge of the PUs' signal [24]. Cyclostationary feature detection is an effective spectrum sensing technique for the detection of very weak signals in the background of noise. Its major drawback however is that it has high computational complexity and it requires a long sensing time due to the longer observation time needed in order to identify the periodic patterns [11]. Wavelet transform detection method requires high sampling rate. This results in high complexity and power consumption. Waveformbased detection is highly robust, due to coherent processing that arises from utilizing deterministic signal components. Its main drawback, however, is that it requires prior information of the PU signals and is susceptible to synchronization errors.

\section{3) Challenges of Spectrum Sensing}

\section{a) Hidden Node Problem}

Due to the effects of multipath fading and shadowing, a CR user can at times not be able to differentiate between a deeply faded and an idle band. This can lead to what is commonly known as the hidden primary user problem. This is experienced when a SU scans for a transmitting PU but cannot correctly predict its presence. CR devices then transmit causing undesired interference to the PU as the primary transmitter's signal could not be correctly detected. Cooperative sensing has been proposed as a means to deal with the hidden primary user problem [25].

\section{b) Sensing Period}

Since PUs have a right to claim their frequency bands at any time, even while being used by the cognitive users, it is paramount that sensing to be carried out as often as possible to 
prevent any possible interference to the PUs. CR users should therefore be able to identify the reappearance of PUs as soon as they reappear and vacate the band immediately. In order to do this, sensing algorithms should have a short but frequent sensing time period in order to avoid co-channel interference. This condition presents a constraint on the quality of sensing (QoS) performance of a sensing algorithm since CR users must frequently interrupt their data transmissions in order to perform spectrum sensing. Consequently, the selection of sensing parameters is constrained as there must be a continuous tradeoff between the sensing period duration and the reliability of sensing.

\section{c) Primary User Emulation Attacks}

In a cognitive radio network, a selfish or greedy SU can mask its air interface to resemble that of a PU in order to gain advantage over other cognitive radio users in what is known as a PU emulation (PUE) attack. This can lead to misleading spectrum sensing results at the expense of legitimate SUs. A solution is to maintain a database of all PUs together with a signature public key encryption value for validating PU transmissions. This will prevent malicious cognitive users from masquerading as PUs. Legitimate PUs would then be required to transmit their transmission together with the encrypted signature value.

\section{d) Spread Spectrum Sensing}

Some PUs employ spread spectrum signaling which is very hard to sense as the signal power is dispersed over a wide frequency range although the actual information bandwidth maybe much narrower. This can be eliminated if the hopping pattern is known so that precise synchronization to the signal is achieved. It can also be solved by using UWB sensing techniques.

\section{e) Receiver Uncertainty Problem}

In transmitter detection mechanisms, CR users are only concerned with information about the primary transmitter but have zero information about the primary receiver. A cognitive radio may therefore be located outside the transmitting range of a PU transmitter but is within a radius of a primary receiver receiving information from the PU. Since it does not sense the presence of any transmitter, it might cause interference to the primary receiver if it tries to transmit. This may lead to interference and is known as the receiver uncertainty problem. A solution to this would be to exploit the local oscillator (LO) leakage power emitted from the RF front-end of the primary receiver to sense it. However, the LO leakage signal is in most cases noted to be very weak [22, 26, 27].

\section{B. Cooperative Sensing}

Individual node sensing is in most cases not sufficiently accurate for sensing the PU signal, due to fading, shadowing, and receiver uncertainty [28]. However, using cooperative sensing, SUs can take advantage of the spatial diversity of each user to co-operate and share the collected sensing information so that the chances of incorrect detection are minimized. Cooperative sensing is employed to improve the detection accuracy of transmitter detection mechanisms through cooperation among SUs on spectrum sensing thereby providing a solution to most issues that come about in spectrum sensing due to noise uncertainty, fading, and shadowing [29, 30]. It also lowers the probabilities of false alarm and misdetection considerably. Additionally, cooperation can help eliminate the hidden PU problem, hence decreasing sensing time. Results have indicated that collaborative sensing results in significantly higher spectrum capacity gains when compared to local sensing. This happens mainly because, in individual node sensing, the CR acts without any information about the PUs position, hence degrading its sensing performance. However, in collaborative spectrum sensing, each of the collaborating CRs observes its own independent fading or shadowing and then these results are combined to paint a better picture of the presence of the PUs. In order to coordinate the collaboration in cooperative sensing architectures, a control channel is required. The control channel can either be implemented using a dedicated band, an unlicensed band e.g. ISM, or an underlay system e.g. ultra-wide band (UWB). The control channel can either be used to share the results of spectrum sensing or for sharing information about the channel allocation amongst the cognitive users [31]. The biggest weakness of cooperative sensing is its augmented delay since data has to be collected from all the cooperating nodes before a decision is made. Cooperation can be executed in two ways: centralized or distributed.

\section{1) Centralized Sensing}

In this kind of cooperation, a central unit called fusion center collects sensing data from the sensing cognitive nodes, identifies an available spectrum hole, and broadcasts this information to the other CRs or uses this information to directly control CR traffic. Figure 8 shows how centralized sensing operates.

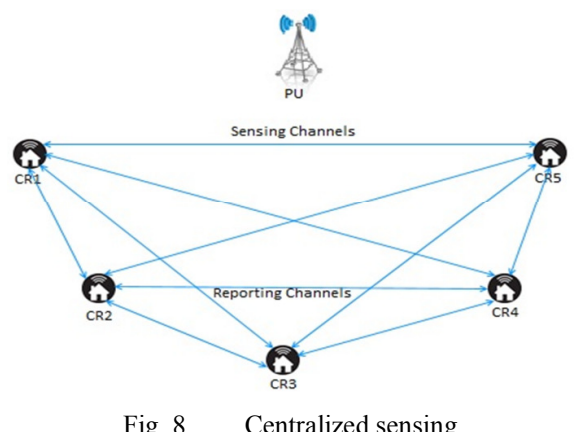

Fig. 8. Centralized sensing

There are two main approaches in centralized sensing.

\section{a) Data Fusion}

With this approach, all SUs send their entire raw sensing data, to the fusion center. CR users only collect the information but do not make any decision. It is the fusion center that determines the band status based on the sensing data received. Sensing data includes the received signal power and the GPS. A drawback associated with this approach is that, in case of a huge number of $\mathrm{CR}$ devices, the bandwidth required for reporting is very large. Data fusion is also referred to as soft combining.

\section{b) Decision Fusion.}

With this approach, each CR user processes its sensing data 
locally and makes a decision on the absence or presence of a PU. This decision is then relayed to the fusion center in form of binary data indicating the band status. The fusion center makes a final decision based on the received decisions from the individual $\mathrm{CR}$ users. The decision is done through a voting scheme. This approach requires less overhead than the data fusion approach as it does not send the entire sensed data to the fusion center.

\section{2) Distributed Sensing}

In distributed cooperative sensing, individual cognitive nodes share sensing data amongst themselves but make individual decisions regarding which spectrum band to use. Distributed sensing scheme is considered superior to centralized sensing as it does not need the deployment of a large-scale infrastructure thus has reduced deployment cost. There are two main approaches in distributed sensing.

\section{a) Distributed Coordinated Techniques}

In this type of coordination neighboring SUs exchange local sensing results. After receiving the sensing results from the others, a SU can make its own judgment regarding the status of a spectrum band, basing it on its own sensing results and the data received from the other SUs using a local criterion and without having the need of a controller (Figure 9).

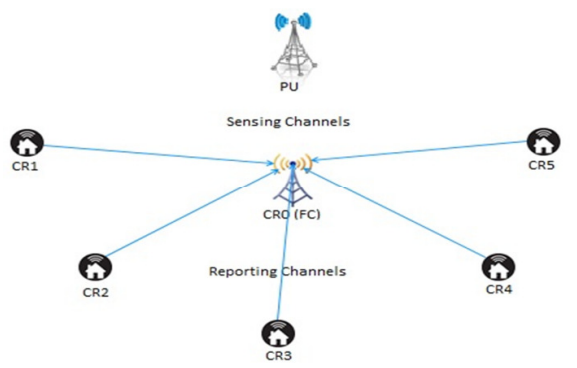

Fig. 9. Distributed coordinated sensing

\section{b) Distributed Uncoordinated Technique}

In some cases, a cognitive user may be adequately able to sense vacant bands but has a very weak reporting channel to the fusion center while another cognitive user within the same user's vicinity has a weak ability to sense vacant channels but a strong reporting channel. In such an instance, both nodes can cooperate: when one senses the vacant, it sends its decision to the neighboring cognitive user which then transmits the information to the fusion center. Therefore, the SU behaves like a relay and the approach becomes a multiple-hop scheme. This technique is thus usually referred to as relay assisted cooperative sensing $[32,33]$ as shown in Figure 10.

\section{Interference Based Detection}

Interference based detection works like a spectrum underlay (UWB) technology, where SUs coexist and transmit simultaneously in the same spectrum band with PUs but only transmit at extremely low power as determined by the interference temperature level in order to curtail any harmful interference to the PUs [32]. There are two main approaches to interference based detection.

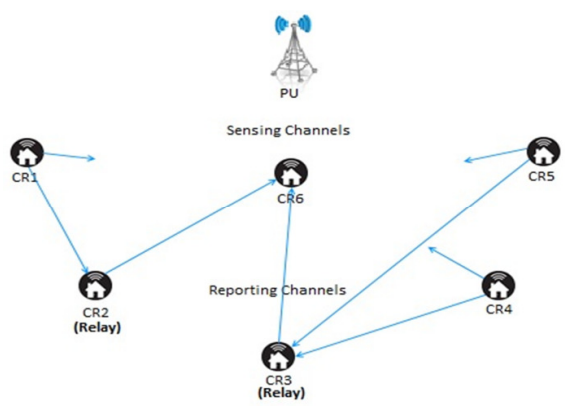

Fig. 10. Distributed uncoordinated sensing

\section{1) Primary Receiver Detection}

In communications, whenever a receiver is receiving data from a transmitter, it usually emits a leakage power from its RF front end. The leakage power is referred to as LO leakage power. It is this leakage power that is exploited to find a PU signal by installing a low cost sensor node close to a PU's receiver. The sensor then reports the sensed LO leakage power emitted by a given RF front end of the PU's receiver within the communication range of a CR [34].

\section{2) Interference Temperature Management}

This approach aims at measuring the interference at the receiver. It does this by setting an upper interference limit for a particular frequency band in specific geographic locations such that CR users are prohibited from causing harmful interference while using the specific band in a given area. Usually, CR transmitters regulate their interference by regulating their out of band emissions (transmission power) based on their positions with respect to PUs $[6,34]$. Thus, CR users do not carry out spectrum sensing. They transmit concurrently with the PUs as long as they are within the specified preset power mask. They can never at any time relay their data with higher power than the preset power even if any PU is absent as they are prohibited to do so. Accordingly, CR users are required to at all times be aware of the location and corresponding upper level of allowed transmit power levels so as to never interfere with the PU transmissions [6].

\section{CONCLUSION}

Current spectrum licensing policy has led to most of the spectrum being assigned to various services. Recent technological developments have led to the development of many new wireless services. These new services require spectrum but were never factored in when the spectrum was first allocated. The spectrum policy is therefore not in tandem with the current needs. Since spectrum is a fixed resource, a solution must be found to accommodate the new and emerging services within the existing spectrum. Studies showed that most of the allocated spectrum is actually underutilized. CR has emerged as an alternative to exploit the underutilized spectrum more efficiently by enabling opportunistic spectrum usage. It has recently received widespread research interest. The CR approach is based on the cardinal rule of being able to correctly sense the available spectrum opportunities.

This paper focused on various spectrum sensing mechanisms used by CRs. The dynamic spectrum nature of 
CRs, where they access different frequency bands, anytime and anywhere, requires particular attention as it helps in spectrum sharing amongst primary and secondary users, thus ensuring there is efficient utilization of all available spectrum. The dynamic spectrum access solves some problems like spectrum scarcity, allowing the exploitation of new opportunities, but on the other hand creates new challenges for spectrum sensing. Analysis showed that there is no perfect sensing mechanism and selecting the best one to use is a delicate process involving tradeoffs between specific requirements. Due to the large range of frequency bands that needs to be explored and sensed to find spectrum holes, CR signal processors need to be faster, more accurate and robust while consuming low power in order to improve the battery life of cognitive devices.

\section{REFERENCES}

[1] S. S. Somawanshi, G. A.Varade, J. M. Mhase, "Cognitive Radio: An Intelligent Wireless Communication System", International Journal of Innovative Research in Science, Engineering and Technology, Vol. 5, No. 3, pp. 3820-3828, 2016

[2] P. Yadav, S. Chatterjee, P. P. Bhattacharya, "A Survey on Dynamic Spectrum Access Techniques in Cognitive Radio", International Journal of Next-Generation Networks, Vol. 4, No. 4, pp. 27-46, 2012

[3] F. R. Yu, Cognitive Radio Mobile Ad Hoc Networks, Springer, 2011

[4] I. J. Mitola, Cognitive Radio Architecture: The Engineering Foundations of Radio XML, John Wiley \& Sons, 2006

[5] Z. Htike, C. S. Hong, "Overview of 802.22 WRAN Standard and Research Challenges", OSIA Standards \& Technology Review, Vol. 24, No. 2, pp. 57-64, 2011

[6] M. Subhedar, G. Birajdar, "Spectrum Sensing Techniques in Cognitive Radio Networks: A Survey", International Journal of Next-Generation Networks, Vol. 3, No. 2, pp. 37-51, 2011

[7] G. Ghosh, P. Das, S. Chatterjee, "Cognitive Radio And Dynamic Spectrum Access - A Study", International Journal of Next-Generation Networks, Vol. 6, No. 1, pp. 43-60, 2014

[8] N. I. Sarkar, "Spectrum Handoff Management in Cognitive Radio Networks: Solutions, Modeling, and Future Research", 11th International Conference on Wireless Networks, Las Vegas, USA, July 16-19, 2012

[9] P. P. Bhattacharya, R. Khandelwal, R. Gera, A. Agarwal, "Smart Radio Spectrum Management for Cognitive Radio", International Journal of Distributed and Parallel Systems, Vol. 2, No. 4, pp. 12-24, 2011

[10] P. K. Verma, S. Taluja, R. L. Dua, "Performance analysis of Energy detection, Matched filter detection \& Cyclostationary feature detection Spectrum Sensing Techniques", International Journal Of Computational Engineering Research, Vol. 2, No. 5, pp. 1296-1301, 2012

[11] R. U. Pal, P. R. Indurkar, P. R. Lakhe, "Review of Performance of Energy Detection, Matched Filter Detection and Cyclostationary Detection Based Spectrum Sensing Under Different Wireless Channels", International Journal of Engineering Research, Vol. 3, No. 2, pp. 6-9, 2015

[12] K. Nirajan, S. Sudeep, S. Suman, B. Lamichhane, "Performance Comparison of Energy Detection Based Spectrum Sensing for Cognitive Radio Networks", International Refereed Journal of Engineering and Science, Vol. 4, No. 8, pp. 1-7, 2015

[13] R. Gill, A. Kansal, "Comparative Analysis of the Spectrum Sensing Techniques Energy Detection and Cyclostationary Feature Detection”, International Journal of Advanced Research in Electrical, Electronics and Instrumentation Engineering, Vol. 3, No. 7, pp. 10601-10608, 2014

[14] G. Nautiyal, R. Kumar, "Spectrum Sensing In Cognitive Radio Using Matlab", International Journal of Engineering and Advanced Technology, Vol. 2, No. 5, pp. 529-532, 2013

[15] H. Saggar, D. K. Mehra, "Cyclostationary Spectrum Sensing in Cognitive Radios Using FRESH Filters", Advances in Wireless Cellular
Telecommunications: 1st ICEIT National Conference on Technologies \& Services, New Delhi, India, April 14-15, 2011

[16] R. V. Babak Ahsant, "A Review of Cooperative Spectrum Sensing in Cognitive Radios", in: Advancement in Sensing Technology: New Developments and Practical Applications, Springer, 2012

[17] R. Garg, N. Saluja, "Spectrum Sensing in Cognitive Radio: Components and Methodologies", World Congress on Engineering and Computer Science, San Francisco, USA, October 19-21, 2016

[18] D. B. Rawat, G. Yan, "Spectrum Sensing Methods and Dynamic Spectrum Sharing in Cognitive Radio Networks: A Survey", International Journal of Research and Reviews in Wireless Sensor Networks, Vol. 1, No. 1, pp. 1-13, 2011

[19] D. L. Chaitanya, K. Manjunatha Chari, "A Review on Different Spectrum Sensing Methods in Cognitive Radio Networks", Paripex Indian Journal of Research, Vol. 4, No. 5, pp. 248-251, 2015

[20] T. Yucek, H. Arslan, “A Survey of Spectrum Sensing Algorithms for Cognitive Radio Applications", IEEE Communication Surveys \& Tutorials, Vol. 11, No. 1, pp. 116-130, 2009

[21] K. Sithamparanathan, A. Giorgetti, Cognitive Radio Techniques: Spectrum Sensing, Interference Mitigation, and Localization, Artech House, 2012

[22] M. B. Dave, Spectrum Sensing in Cognitive Radio: Use of CycloStationary Detector, MSc Thesis, National Institute of Technology, Rourkela, India, 2012

[23] P. Kaur, C. Singh, "Spectrum Sensing based on Energy Detection in Cognitive Radio", International Journal of Advanced Research in Electronics and Communication Engineering, Vol. 4, No. 5, pp. 12141217,2015

[24] R. Kumar, "Analysis of Spectrum Sensing Techniques in Cognitive Radio", International Journal of Information and Computation Technology, Vol. 4, No. 4, pp. 437-444, 2014

[25] C. S. Sum, G. P. Villardi, M. A. Rahman, T. Baykas, H. N. Tran, Z. Lan, C. Sun, Y. Alemseged, J. Wang, C. Song, C. W. Pyo, S. Filin, H. Harada, "Cognitive communication in TV white spaces: An overview of regulations, standards, and technology", IEEE Communications Magazine, Vol. 51, No. 7, pp. 138-145, 2013

[26] I. F. Akyildiz, W. Y. Lee, M. C. Vuran, S. Mohanty, “A Survey on Spectrum Management in Cognitive Radio Networks", IEEE Communications Magazine, Vol. 46, No. 4, pp. 40-48, 2008

[27] D. Teguig, B. Scheers, V. Le Nir, "Data Fusion Schemes for Cooperative Spectrum Sensing in Cognitive Radio Networks", 2012 Military Communications and Information Systems Conference, Gdansk, Poland, October 8-9, 2012

[28] O. Fatemieh, R. Chandra, C. A. Gunter, "Low Cost and Secure Smart Meter Communications using the TV White Spaces", 3rd International Symposium on Resilient Control Systems, Idaho Falls, USA, August 1012,2010

[29] Y. L. Lee, W. K. Saad, A. A. El-Saleh, M. Ismail, "Improved Detection Performance of Cognitive Radio Networks in AWGN and Rayleigh Fading Environments", Journal of Applied Research and Technology, Vol. 11, No. 3, pp. 437-446, 2013

[30] Y. Zhang, R. Yu, M. Nekovee, Y. Liu, S. Xie, S. Gjessing, "Cognitive Machine-to-Machine Communications: Visions and Potentials for the Smart Grid”, IEEE Network, Vol. 26, No. 3, pp. 6-13, 2012

[31] C. Xin, M. Song, "Opportunistic Spectrum Access", in: Spectrum Sharing for Wireless Communications, pp. 7-16, Springer, 2015

[32] G. Ghosh, P. Das, S. Chatterjee, "Simulation and Analysis of Cognitive Radio System using Matlab", International Journal of Next-Generation Networks, Vol. 6, No. 2, pp. 31-45, 2014

[33] D. D. Kulkarni, D. Patel, V. P. Gejji, "Spectrum Sensing Techniques and Dynamic Spectrum Allocation", International Journal of Innovative Research in Electrical, Electronics, Instrumentation and Control Engineering, Vol. 4, No. 4, pp. 103-106, 2016

[34] R. M. S. Venkateswari, “An Overview of Cognitive Radio Architecture A Review", Journal of Theoretical and Applied Information Technology, Vol. 41, No. 1, pp. 20-25, 2012 